\title{
SOLITONS IN A COMPRESSIBLE HEISENBERG CHAIN
}

\author{
S. LIPIŃSKI \\ Institute of Molecular Physics, Polish Academy of Sciences \\ Smoluchowskiego 17/19, 60-179 Poznań, Poland
}

Nonlinear excitations in a Heisenberg chain coupled to the lattice in the presence of an external field are studied within a semi-classical approach.

PACS numbers: $75.10 . \mathrm{Jm}, 75.40 . \mathrm{Gb}$

The problem of a soliton formation in the Heisenberg chain coupled to the lattice has been considered both for the classical [1-4] and for the quantum model [5]. In spite of the fact that nonlinearity resulting from the magnon-magnon interactions leads to the soliton solutions itself this contribution has been completely neglected in the previous quantum approach to the solitary magnon lattice wave [5].

The aim of the present paper is to discuss how the magnetic solitons having the sense of the multi-magnon bound states [6] are influenced by the spin-phonon coupling.

We consider the compressible Heisenberg chain

$$
\begin{aligned}
H= & \sum_{i} \frac{p_{i}^{2}}{2 m}+\frac{m v_{0}^{2}}{2} \sum_{i}\left(u_{i+1}-u_{i}\right)^{2}-J \sum_{i} S_{i} S_{i+1} \\
& -\mu h_{0} \sum_{i} S_{i}^{z}-g \sum_{i}\left(u_{i+1}-u_{i}\right) S_{i} S_{i+1},
\end{aligned}
$$

where $u$ is the displacement operator of the magnetic ion from its equilibrium, $p$ - the momentum operator, $v_{0}$ - the sound velocity, $g$ is the exchange striction coefficient $(g=\partial J / \partial x)$ and $h_{0}$ is the external magnetic field. Here we assume the lattice constant to be equal to one. We take the Holstein-Primakoff transformation of the spin operators in the low temperature approximation

$$
\begin{aligned}
& S^{+}(i) \approx(2 S)^{1 / 2}\left[a(i)-a^{+}(i) a(i) a(i) / 4 S\right], \\
& S^{-}(i) \approx(2 S)^{1 / 2}\left[a^{+}(i)-a^{+}(i) a^{+}(i) a(i) / 4 S\right], \\
& S^{z}(i)=S-a^{+}(i) a(i) .
\end{aligned}
$$

We rewrite $H$ using the boson representations (2) restricted to the biquadratic terms in the Heisenberg Hamiltonian and to the bilinear in the spin-lattice coupling. The latter approximation is justified by the assumption that the spin-phonon 
coupling is much smaller than the spin-spin interaction. The approximate Hamiltonian reads

$$
\begin{aligned}
H= & E_{0}+H_{\mathrm{L}}+\left(\mu h_{0}+J S\right) \sum_{i} a^{+}(i) a(i)+\frac{J S}{2} \sum_{i \delta}\left[a^{+}(i+\delta) a(i+\delta)\right. \\
& \left.-a^{+}(i) a(i+\delta)-a^{+}(i+\delta) a(i)\right]-\frac{1}{2} \sum_{i \delta} a^{+}(i) a(i) a^{+}(i+\delta) a(i+\delta) \\
& +\frac{J}{2} \sum_{i \delta}\left[a^{+}(i+\delta) a^{+}(i+\delta) a(i+\delta) a(i)+a^{+}(i+\delta) a^{+}(i) a(i) a(i)\right. \\
& \left.+a^{+}(i) a^{+}(i+\delta) a(i+\delta) a(i+\delta)+a^{+}(i) a^{+}(i) a(i) a(i+\delta)\right] \\
& +g S \sum_{i}\left(u_{i+1}-u_{i}\right)\left[a^{+}(i+1) a(i+1)+a^{+}(i) a(i)\right],
\end{aligned}
$$

where $H_{\mathrm{L}}$ denotes the lattice part of $(1)$ and $E_{0}$ is the ground state energy. Let us introduce the multi-mode coherent states defined by

$$
\begin{aligned}
& |\alpha\rangle=\exp \sum_{i}\left(\alpha_{i} a_{i}^{+}-\alpha_{i}^{*} a_{i}\right)|0\rangle_{\mathrm{F}}, \\
& |\Delta, \Pi\rangle=\exp \left[-\frac{\mathrm{i}}{\bar{\hbar}} \sum_{i}\left(\Delta_{i} p_{i}-\Pi_{i} u_{i}\right)\right]|0\rangle_{\mathrm{LATT}},
\end{aligned}
$$

where $|0\rangle_{F}$ denotes a ferromagnetic ground state and $|0\rangle_{\text {LATT }}$ - the lattice vacuum. In the following the functional $F(t)=\langle\Pi, \Delta, \alpha|H| \alpha, \Delta, \Pi\rangle$ serves us as a Hamilton functional. We look for the solutions of the Hamilton equations (5) in the continuum approximation because we are interested in the excitations of space extension much larger than the lattice constant

$$
\begin{aligned}
& \mathrm{i} \hbar \frac{\partial \alpha_{i}}{\partial t}=\frac{\partial F}{\partial \alpha_{i}^{*}}, \\
& \frac{\partial \Delta_{i}}{\partial t}=\frac{\partial F}{\partial \Pi_{i}}, \quad \frac{\partial \Pi_{i}}{\partial t}=\frac{-\partial F}{\partial \Delta_{i}} .
\end{aligned}
$$

Moreover, we consider only the stationary profile solutions

$$
\Delta=\Delta(\xi), \quad \alpha=\alpha_{0}(\xi) \exp (\mathrm{i} \Theta(x, t)), \quad \xi=x-v t .
$$

Having in mind the above assumptions one gets from (5) the nonlinear Schrödinger-like equation

$$
\begin{aligned}
\mathrm{i} \hbar \frac{\partial \alpha}{\partial t} & =\mu h_{0} \alpha-J S \frac{\partial^{2} \alpha}{\partial x^{2}}-B|\alpha|^{2} \alpha-J \alpha\left|\frac{\partial \alpha}{\partial x}\right|^{2} \\
& -\frac{1}{2} J \alpha^{2} \frac{\partial^{2} \alpha^{*}}{\partial x^{2}}+\frac{1}{2} J \alpha^{*}\left(\frac{\partial \alpha}{\partial x}\right)^{2}
\end{aligned}
$$

where

$$
B=\frac{(2 g S)^{2}}{m\left(v_{0}^{2}-v^{2}\right)}
$$

and the lattice deformation reads

$$
\frac{\partial \Delta}{\partial x}=\frac{-2 g S}{m\left(v_{0}^{2}-v^{2}\right)}|\alpha(x, t)|^{2} .
$$


The one-soliton solution to Eq. (7) is known [7] and is given by

$$
\begin{aligned}
\alpha_{0}^{2}(\xi) & =\frac{4 \mu h_{0} \beta^{2}}{J} /\left[\Omega+2 \gamma+\left(\Omega^{2}+\gamma V^{2}\right)^{1 / 2} \cosh \left(L^{-1} \xi\right)\right], \\
\Theta(\xi) & =\frac{1}{2} V \xi \\
+\arctan & {\left[\left(\frac{\left.\Omega^{2}+\gamma V^{2}\right)^{1 / 2}+\left(\Omega-V^{2} / 2\right)}{\left(\Omega^{2}+\gamma V^{2}\right)^{1 / 2}-\left(\Omega-V^{2} / 2\right)}\right)^{1 / 2} \tanh \left(L^{-1} \xi / 2\right)\right], }
\end{aligned}
$$

where

$$
\begin{aligned}
& \gamma=B / J, \quad \Omega=\mu h_{0} / J S-\gamma, \quad V=\hbar v / J S, \\
& \beta^{2}=1-V^{2} / 4(\Omega+\gamma), \quad L^{-1}=2 \beta\left(\mu h_{0} / J S\right)^{1 / 2} .
\end{aligned}
$$

The corresponding total energy and the local magnetization distribution $\left\langle S^{z}\right\rangle=$ $S-\left\langle\alpha\left|a^{+} a\right| \alpha\right\rangle$ are

$$
\begin{aligned}
E= & 4 J S^{2} \beta\left(\frac{\mu h_{0}}{J S}\right)^{1 / 2} \\
& +\frac{4 S \mu h_{0}}{\gamma^{1 / 2}} \operatorname{arctanh}\left(\frac{\Omega+2 \gamma-\left(\Omega^{2}+\gamma V^{2}\right)^{1 / 2}}{\Omega+2 \gamma+\left(\Omega^{2}+\gamma V^{2}\right)^{1 / 2}}\right)^{1 / 2}, \\
\left\langle S^{z}\right\rangle= & S-\frac{4 \mu h_{0}}{J} \beta^{2} /\left[\Omega+2 \gamma+\left(\Omega^{2}+\gamma V^{2}\right)^{1 / 2} \cosh \left(L^{-1} \xi\right)\right] .
\end{aligned}
$$

In the absence of spin-phonon coupling $(\gamma=0)$ our solution is the same as the one obtained classically by Tjon and Wright [8]. The coupling to the lattice broadens the soliton, reduces its amplitude and energy. For small velocities the soliton $(10,11)$ corresponds to the large deviations from the ground state. The energy of the static soliton is of order of $\left(J S / \mu h_{0}\right)^{1 / 2}$ larger than the energy of the one-magnon state. For large velocities close to the critical $\left(V_{c}=2\left(\mu h_{0} / J S\right)^{1 / 2}\right)$ the configuration $(10,11)$ comes close to the ground state. The case of the magnetic Davydov type solitons, energetically more favorable than magnons, were previously discussed by Pushkarov [5]. In the present formalism these solutions can be obtained by considering only the first three terms of the right hand side of Eq. (7). It corresponds to the assumption of large space extension of the excitations. The influence of the magnon-magnon interactions on the Davydov type solitons is under consideration and will be published elsewhere.

The author thanks Professor J. Morkowski for a discussion.

\section{References}

[1] M. Cieplak, L.A. Turski, J. Phys. C, Solid State Phys. 13, L777 (1980).

[2] J. Fivez, J. Phys. C, Solid State Phys. 15, L641 (1982).

[3] E. Magyari, J. Phys. C, Solid State Phys. 15, L1159 (1982).

[4] M.J. Skrinjar, S.D. Stojanovič, D.V. Kapor, J. Phys. C, Solid State Phys. 19, 5885 (1985). 
[5] D.I. Pushkarov, Kh.I. Pushkarov, Phys. Status Solidi B 81, 703 (1977).

[6] L.G. de Azevedo, M.A. de Moura, C. Cordeiro, B. Zeks, J. Phys. C, Solid Phys. 15, 7391 (1982).

[7] O.F. de Alcantara, M.A. de Moura, Phys. Lett. A 94, 239 (1983).

[8] J. Tjon, J. Wright, Phys. Rev. B 15, 3470 (1977). 